LA-UR-03-3082

Approved for public release; distribution is unlimited.

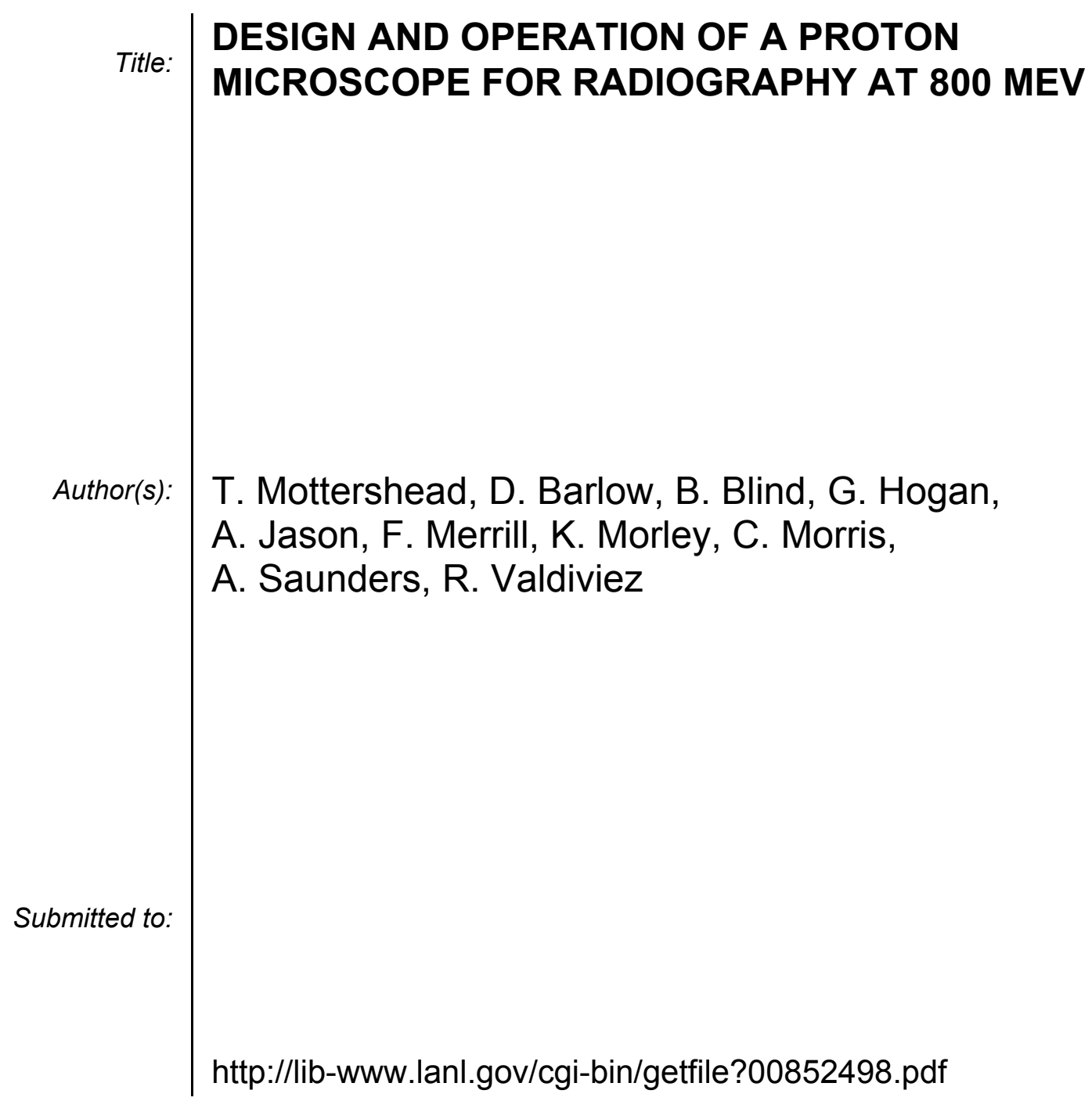




\title{
DESIGN AND OPERATION OF A PROTON MICROSCOPE FOR RADIOGRAPHY AT $800 \mathrm{MEV}$
}

\author{
T. Mottershead, D. Barlow, B. Blind, G. Hogan, A. Jason, F. Merrill, K. Morley, \\ C. Morris, A. Saunders, R. Valdiviez \\ Los Alamos National Laboratory, Los Alamos, NM 87545
}

\begin{abstract}
A high-magnification high-resolution option is desirable for the study of small-scale dynamic experiments at the LANSCE 800-MeV Proton Radiography Facility. Magnification is achievable by either repowering the existing imaging-lens quadrupoles, using new highgradient quadrupoles, or some hybrid combination of the two. The large and complex parameter space of magnetic optics solutions was studied extensively with the 3rd order optics code MARYLIE. Some of the hybrid solutions achieve magnifications up to 150 , but at the price of high chromatic aberrations. In the end, a design using only new high-gradient permanent-magnet quadrupoles was selected and built at the design parameters that minimized chromatic aberration per unit magnification. The design has a moderate magnification of 7.1 and 15.8 at the two existing image stations. First-beam commissioning results exceeded expectations. Image contrast is produced by multiple Coulomb scattering in the thin objects. Early experimental objectives are to optimize this contrast by collimator design and by adjusting the correlation in the illuminating beam, as well as to characterize the (quite high) resolution limits of the system.
\end{abstract}

\section{THE PROTON RADIOGRAPHY MICROSCOPE}

Proton radiography is a new technology for imaging the interior of objects that can be penetrated by high-energy protons. A broad beam of high-energy protons shines through the object, and suffers path-dependent attenuation by collisions with the nuclei. It also suffers pathdependent spread in angle by multiple Coulomb scattering (MCS). The protons exit the object with an assortment of energies due to the losses on the varying paths through the object. The net effect is that an image is imprinted in the exit beam by its passage through the object. A magnetic lens after the object extracts the implicit image by refocusing the varied scattering angles onto a detector plane, where it is recorded by a set of gated TV cameras.

\section{The pRAD System at LANSCE}

The operational proton radiography facility at LANSCE contains a diffuser to spread the incident beam, an illuminator matching section to tailor its phase space distribution, and a monitor lens to copy this distribution onto the object. There are two consecutive magnetic imaging lenses after the object, each with its own image plane, called IL1 and IL2. Each lens is made of two identical cells containing a symmetric doublet of 12Q24 quadrupoles. The magnification is unity. All eight quads (named A through $\mathrm{H}$ in Fig. 1) have the same $5.7 \mathrm{~T} / \mathrm{m}$ gradient, with alternating signs. The proton-microscope project was aimed at improving the resolution of the images for small objects, with the goal of studying phenomena on a scale of some tens of microns. The new equipment was to fit smoothly into the operations at LANSCE, and use as much of the existing equipment (e.g. the camera systems) as possible.

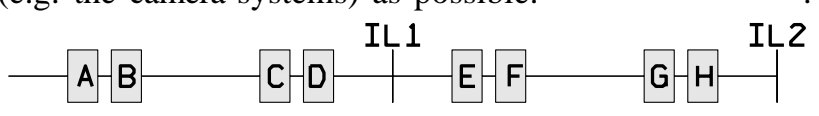

Figure 1 LANSCE pRAD Identity Lens Configuration

\section{Image Resolution}

The overall resolution of the system is determined by the performance of the digital TV readout cameras, by the scattering of light in the detector fluor, and by the performance of the magnetic lens. Magnetic magnification can spread the image over enough pixels to overcome the limitations of the optical system. The magnetic lens maps the phase space coordinates $\left(x_{0}, \theta_{0}\right)$ of the proton exiting the object to final position $x_{f}=R_{11} x_{o}+R_{12} \theta_{0}$ on the detector plane. Focus means the final position is independent of the initial angle $\theta_{0}$. This occurs at the momentum where $R_{12}=0$. Protons with other momenta are out of focus. To first order in $\Delta$, their position on the detector plane is

$$
x_{f}=M x_{0}+\Delta\left(R_{11}^{\prime} x_{0}+R_{12} \theta_{0}\right)
$$

where $M=R_{11}$ is the magnification, $\Delta=\delta p / p$ is the deviation from the focus momentum, and the primes indicate derivatives with respect to $\Delta$. The first thing to note is that rays with the correlation $\theta_{0}=w x_{0}$, where $w=-R_{11}{ }^{\prime} / R_{12}{ }^{\prime}$ are achromatic in that their final position is independent of their energy. An upstream matching section imposes this achromatic correlation on the illuminating beam, so a ray exiting the object with MCS angle $\varphi$ has an initial trajectory angle $\theta_{0}=w x_{0}+\varphi$. Its final position is therefore $x_{f}=M x_{0}+C \varphi \Delta$, where $C \equiv R_{12}$ ' is called the chromatic aberration coefficient of the lens. The position error in object coordinates for an off-energy proton is therefore $-C \varphi \Delta / M$. An appropriate average over the distribution of scattering angles and energy shifts gives the resolution spot size associated with a given path through the object. This makes chromatic aberration per unit magnification the proper measure of microscope resolution. The goal is to improve on the $\mathrm{C} / \mathrm{M}=12$ meters chromatic aberration coefficient of the Identity lens. 


\section{Fourier Points}

The position at longitudinal location $\mathrm{z}$ of the general scattered ray with initial coordinates $\left(x_{0}, w x_{0}+\varphi\right)$ is $x_{0}[C(z)+w S(z)]+\varphi S(z)$, where $S(z)$ and $C(z)$ are the usual sinelike and cosinelike basis rays of magnetic optics. The term in square brackets is the achromatic basis ray. The Fourier point is its zero crossing, where the trajectory position depends only on the MCS angle $\varphi$, independent of the initial position. A collimator at this location can attenuate the scattered rays, providing image contrast by darkening the final image pixels in the locations containing the material causing the scattering. The location and strength of the Fourier point is an important design consideration. It depends on the correlation coefficient $w$ imposed by the matching section, and is not necessarily the same in both planes. Collimators can be optimized for the range of objcct areal densities to be studied.

\section{Field-of-View}

The expected mean scattering angle and energy shift depend on the size and composition of the object. The size of possible objects is limited by the field-of-view (FoV) of the lens, which is in turn limited by either the size of the beam pipe or the size of the detector. A square detector of dimension $\mathrm{L} \times \mathrm{L}$ can record an FoV of dimension L/M. The thickness of the possible object is presumably about the same. We may estimate the nuclear attenuation in pathlength $\mathrm{z}$ through an object as $\exp -\left(z \rho / \lambda_{I}\right)$, where $\rho$ is the density, and $\lambda_{I}$ is the nuclear interaction length in $\mathrm{g} / \mathrm{cm}^{2}$. The standard detector at LANSCE is a 5 glass fluor, so $\mathrm{L}=12.7 \mathrm{~cm}$. With a magnification of 12.7, the FoV would be $1 \mathrm{~cm}$. Higher magnification leads to a smaller FOV, suited for studying smaller objects. Note that nuclear attenuation in $1 \mathrm{~cm}$ of $\mathrm{Cu}$, for example, is only $1 \%$. The RMS polar MCS angle is $13 \mathrm{mrad}$, and the energy loss is $12.6 \mathrm{MeV}$. The MCS angle scales as the square root of the path length divided by the radiation length.

\section{DESIGN OPTIONS}

We seek to modify the operational system to achieve higher magnification and lower specific chromatics. The three basic alternatives are to repower the existing big quadrupoles, to build a new high-gradient small lens with permanent-magnet quadrupoles (PMQs), or to build a hybrid system using both. In all cases, the three design constraints are focus $\left(R_{12}=R_{34}=0\right)$, and equal magnification $\left(R_{11}= \pm R_{33}\right)$ in both planes.

\section{Repowered Big Quadrupoles}

Three constraints for eight quadrupoles allows a large space of allowed solutions. A methodical survey of this parameter space was undertaken, including analytic studies in the thin-lens approximation. The simplest example would be searching for a magnifier solution for a single four-quad lens. One of the quads could be set arbitrarily, and the other three varied to fit the design constraints. The scanning-the-fit procedure in the $3^{\text {rd }}$ order design code MARYLIE was used to compute the relative chromatic aberration for all possible values of the fourth quad. The result was a peak magnification of $M=2.265$ on IL1, with relative chromatics of $3 \mathrm{~m}$ in the $\mathrm{x}$-plane, and $19 \mathrm{~m}$ in the y-plane. A compound lens with two of these in succession results in squaring the magnification to $\mathrm{M}=5.131$ on IL2, with chromatics of 3.5 and 22.7. Another four quad solution in the pattern $\mathrm{AB} 00 \mathrm{E} 00 \mathrm{H}(0$ marks a quad that is turned off) results in peak magnification of $\mathrm{M}=5.82$ and chromatics of 2.6 and 15.5 . A pattern of the form $\mathrm{ABCBAGH0}$, with $\mathrm{B}=-\mathrm{A}$, went to a magnification of $\mathrm{M}=33$, with chromatics of 7.07 and 32.25. Here quad $\mathrm{H}$ was scanned, and quads $\mathrm{A}, \mathrm{C}$, and $\mathrm{G}$ adjusted to fit the 3 design constraints. Fig. 2 shows the relative chromatic aberration in both planes as a function of magnification for the later two designs. In all cases the relative chromatics are worse in at least one plane than the original Identity lens .

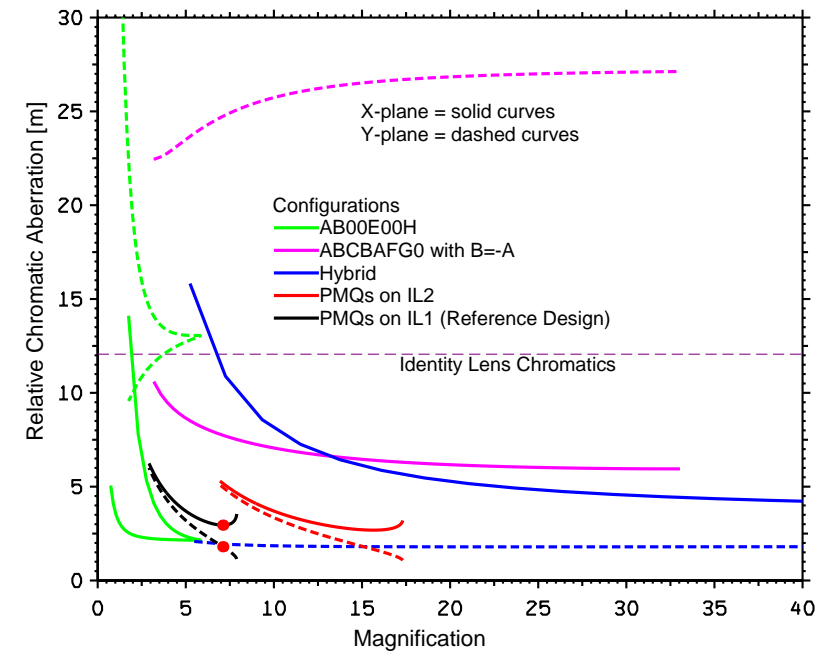

Figure 2. Parametric curves of relative chromatic aberration vs. magnification for 5 cases

\section{High-Gradient PMQs}

A Russian quadruplet has an inner doublet inside an outer doublet of opposite sign and half the strength. This type of lens made with high gradient PMQs works very well. The other big quadrupoles are turned off, and merely serve as a drift space. The proton trajectories from the object to the first image plane are shown in Fig 3.



Figure 3: Rays in the PMQ microscope 
The magnification depends on the distance from the object to the first quadrupole. The performance curves in Fig. 2 show an optimum at a focal standoff of $40 \mathrm{~cm}$, which was chosen for the actual device. Since the strength of the quadrupoles is fixed, the lens must be focused by moving them. The rays in fig 3 are color coded by scattering angle in 3 milliradian increments, showing the Fourier point between the second and third quadrupoles.

\section{Hybrid Systems}

The PMQ lens was designed to operate with the 6 big downstream quads turned off. Solutions with the downstream quads turned on, however, can produce very high magnifications of over 100. The FOV is correspondingly small, while the relative aberrations are somewhat worse than with the PMQ lens alone. This may be a useful configuration for some experiments.

\section{CONSTRUCTION AND OPERATION}

The design iterations led to the final choice of 1.5 inch thick PMQ segments, with an inner diameter of 1.375 inches and an outer diameter of 4.0 inches. With the $1.2 \mathrm{~T}$ remnant field of $\mathrm{NeFeB}$, the gradient is $71.6 \mathrm{~T} / \mathrm{m}$. The inner of quadrupoles are assembled from 4 segments each, so are 6 inches long. The outer half-strength pair are 3 inches long, and made of 2 segments each. The multipole error tolerances were determined in a study of their effect on image quality.

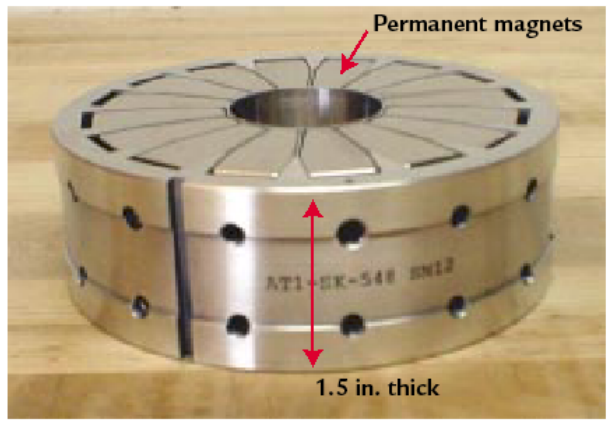

Figure 4. A PMQ segment.

The PMQs were made by Aster Corporation in 1.5 inch thick segments shown in fig 3. The segments were measured when received, and were well within design specifications. The measured gradient averaged $72.83 \mathrm{~T} / \mathrm{m}$. The microscope is mounted in the area $\mathrm{C}$ beamline in the location normally occupied by the first two large quadrupoles, which have been mounted on rails to facilitate the configuation change (fig 5). The quads slide over the 1.25" stainless steel beam pipe. Their position may be adjusted from the control room by actuators to focus the lens on either IL1 with a magnification of 7.2, or IL2 with a magnification of 15.8. The matching section was set to illuminate the object with the achromatic rays demanded by the lens. This placed the Fourier points about $12 \mathrm{~mm}$ apart and $6 \mathrm{~cm}$ before the entrance of the third quad. A tungsten collimator with a simple 5/32" aperture was placed in the pipe at this location.

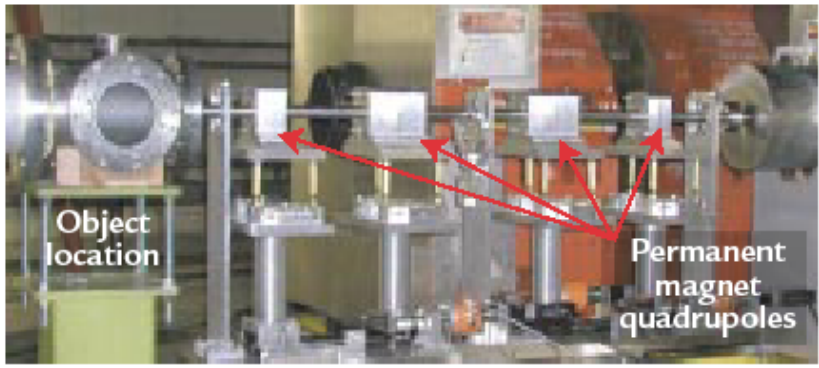

Figure 5. The microscope in the beamline. Large red objects in background are big quads $\mathrm{A}$ and $\mathrm{B}$.

\section{First Beam Test}

The first test object used was a small stainless steel plate with the word LANL milled in it in $7 \mathrm{~mm}$ high letters.

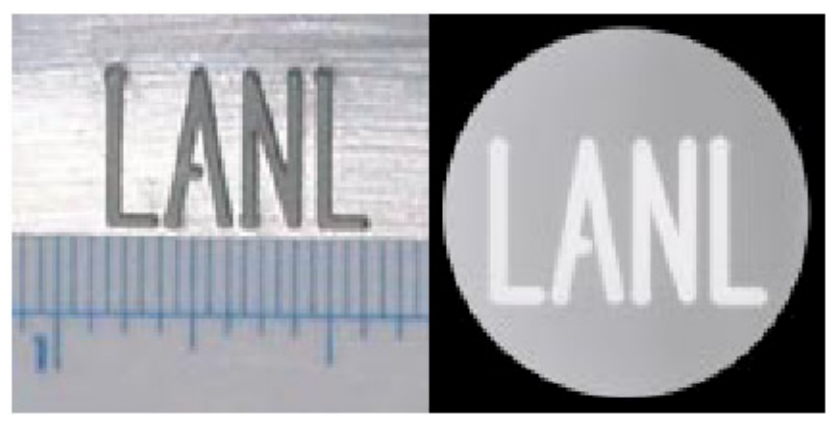

Figure. 6. First test object. Finest tick marks on ruler are $1 / 32$ inch. Proton radiograph on right

At first beam a good picture was obtained with the nominal design settings. After some tuning, fig 6 was obtained.

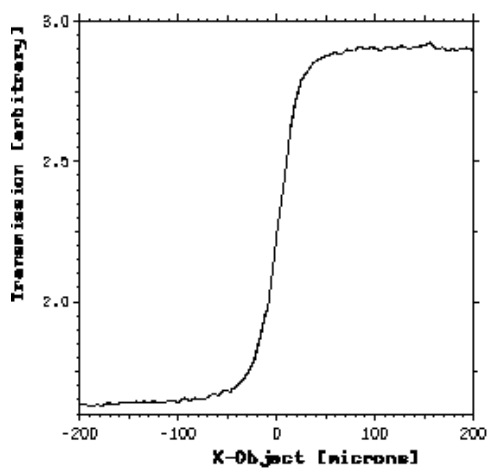

Figure. 7 Profile through edge of a letter

Fig. 7 shows a profile through the edge of a letter. Analysis of this profile indicates an overall resolution of about 17 microns, including the optical system. A finer test object is needed to fully characterize the magnetic resolution of the system, which is expected to be somewhat better.

\section{REFERENCES}

[1] C. T. Mottershead and J. D. Zumbro, "Magnetic Optics for Proton Radiography”, Proc. 1997 Particle Accelerator Conf, Vancouver 1397 (1997)

[2] G. E. Hogan et al. "Proton Radiography", Proc. 1999 Particle Accelerator Conf, New York 579 (1999) 